九州大学学術情報リポジトリ

Kyushu University Institutional Repository

Simultaneous Enhancement in the Electrical Conductivity and Reduction in the Lattice Thermal Conductivity Leading to Enhanced Thermoelectric ZT Realized by Incorporation of Metallic Nanoparticles into 0xide Matrix

Hirata, Shinji

Department of Applied Science for Electronics and Materials, Interdisciplinary Graduate School of Engineering Sciences, Kyushu University

Ohtaki, Michitaka

Department of Applied Science for Electronics and Materials, Interdisciplinary Graduate School of Engineering Sciences, Kyushu University

https://doi.org/10.5109/2740934

出版情報 : Evergreen. 7 (1)，pp.1-6，2020-03. 九州大学グリーンテクノロジー研究教育センター バージョン :

権利関係 : 


\title{
Simultaneous Enhancement in the Electrical Conductivity and Reduction in the Lattice Thermal Conductivity Leading to Enhanced Thermoelectric ZT Realized by Incorporation of Metallic Nanoparticles into Oxide Matrix
}

\author{
Shinji Hirata ${ }^{1}$, Michitaka Ohtaki ${ }^{1,2, *}$ \\ ${ }^{1}$ Department of Applied Science for Electronics and Materials, Interdisciplinary Graduate School \\ of Engineering Sciences, Kyushu University, Japan \\ ${ }^{2}$ Transdisciplinary Research and Education Center for Green Technologies, Kyushu University, Japan \\ *Corresponding author, E-mail: ohtaki@kyudai.jp
}

(Received March 9, 2020; Received March 27; 2020; accepted March 27, 2020).

\begin{abstract}
Nanoporous $\mathrm{Fe}_{3} \mathrm{O}_{4}$ composites containing metallic $\mathrm{Cu}$ nanoparticles were synthesized by reducing-leaching treatment of $\mathrm{CuFe}_{2} \mathrm{O}_{4}$ precursor. Thermoelectric properties of the composites sintered at $1270{ }^{\circ} \mathrm{C}$ greatly improved compared with $\mathrm{Fe}_{3} \mathrm{O}_{4}$ without $\mathrm{Cu}$, presumably due to a nanoporous structure and the $\mathrm{Cu}$ nanoparticles remaining in the $\mathrm{Fe}_{3} \mathrm{O}_{4}$ matrix after acid leaching. The electrical and thermal conductivity indicate that $\mathrm{Cu}$ in the matrix promotes the electron conduction and suppresses the phonon conduction, improving the dimensionless figure-of-merit, $Z T$.
\end{abstract}

Keywords: thermoelectrics; nanocomposites; metallic nanoparticles; nanoporous structure; phonon scattering; oxides

\section{Introduction}

Global energy consumption increasing year by year requires a technique development of renewable energy source such as wind and solar energy. ${ }^{1-2)}$ Thermoelectrics, a class of materials utilized to convert heat directly into electricity by the Seebeck effect, is also expected to be another source of renewable energy. Thermoelectric materials are evaluated by the dimensionless figure-ofmerit,

$$
Z T=\frac{S^{2} \sigma}{\kappa} T=\frac{S^{2} \sigma}{\kappa_{\mathrm{el}}+\kappa_{\mathrm{ph}}} T
$$

where $\sigma$ is the electrical conductivity, $\mathrm{S}$ is the Seebeck coefficient, $\mathrm{T}$ is the absolute temperature, $\kappa$ is the thermal conductivity, $\kappa_{\mathrm{el}}$ is the electron thermal conductivity, and $\kappa_{\mathrm{ph}}$ is the phonon thermal conductivity. Good thermoelectric materials should have high $\sigma$ and $S$ with low $\kappa$ simultaneously. However, $\sigma, S$ and $\kappa_{\text {el }}$ are all a function of the carrier concentration and hence interdependent each other, making good materials difficult to develop. On the other hand, $\kappa_{\mathrm{ph}}$ is associated with crystal lattice vibrations, and the only parameter insensitive to the carrier concentration, being independent of other parameters in ZT. Therefore, an approach to selectively decrease $\kappa_{\mathrm{ph}}$ is one of the most prospective way to improve $Z T^{3-20)}$

Recent studies showed that nanostructure control in materials is an efficient way to decrease the phonon thermal conductivity. ${ }^{21)}$ Coarse porous structure can decrease $\kappa$, while $\sigma$ is also suppressed equally or more strongly due to the reduction of the cross-sectional area for the electron conduction. ${ }^{22)}$ Meanwhile, nanocomposite structure can selectively reduce $\kappa$, specifically $\kappa_{\text {ph }}$, because of phonon scattering by nanopores and nanoparticles. ${ }^{23)}$ This specific decrease in $\kappa_{\text {ph }}$ is governed by the composite structure, particularly the size of the pore and the particles.

In this paper, we report novel synthesis of nanoporous oxide containing metallic nanoparticles and investigation of a relation between the structure and the thermoelectric properties of the oxide composites. A copper spinel $\mathrm{CuM}_{2} \mathrm{O}_{4}(\mathrm{M}=\mathrm{Fe}, \mathrm{Al}, \mathrm{Mn}, \mathrm{Ni})$ has been reported to form metallic $\mathrm{Cu}$ nanoparticles in $\mathrm{M}$-oxide matrix by heating in a reducing atmosphere. ${ }^{24-26)}$ If the $\mathrm{Cu}$ nanoparticles in the matrix can be selectively removed, nanosized pores will remain in the oxide. Here, we focused on $\mathrm{CuFe}_{2} \mathrm{O}_{4}$, which is expected to form $\mathrm{Fe}_{3} \mathrm{O}_{4}$ composite containing $\mathrm{Cu}$ nanoparticles after $\mathrm{H}_{2}$ reduction, because the iron oxide is more electrically conductive than aluminum or manganese oxides to be obtained as a matrix by reducing $\mathrm{CuAl}_{2} \mathrm{O}_{4}$ and $\mathrm{CuMn}_{2} \mathrm{O}_{4}$. Finally, we aim to establish an innovative nanostructure-control approach to achieve high thermoelectric performance.

\section{Experimental}

\subsection{Sample preparation}


$\mathrm{CuFe}_{2} \mathrm{O}_{4}$, a precursor of the nanocomposites was prepared by calcining a mixed powder of $\alpha-\mathrm{Fe}_{2} \mathrm{O}_{3}(99 \%$, Kishida Kagaku) and $\mathrm{CuO}\left(99.9 \%\right.$, Wako) at $1000{ }^{\circ} \mathrm{C}$ for $4 \mathrm{~h}$ in air. The copper spinel oxide thus formed was ground in a mortar and heated in a reducing atmosphere $\left(5 \% \mathrm{H}_{2} / \mathrm{N}_{2}\right)$ at $270{ }^{\circ} \mathrm{C}$ for $14 \mathrm{~h}$. After the reduction, the sample powder was washed with $69 \%$ nitric acid for leaching of metallic copper. The reducing and leaching processes (denoted as R-L cycle hereafter) were repeated under the same conditions for some samples. These powders were finally sintered at $1270{ }^{\circ} \mathrm{C}$ for $3 \mathrm{~h}$ in an $\mathrm{N}_{2}$ atmosphere. For comparison, a reagent grade $\mathrm{Fe}_{3} \mathrm{O}_{4}(95 \%$, Wako) was also sintered under the same conditions.

\subsection{Characterization}

Powder x-ray diffraction (XRD) measurements were performed using a PANalytical EMPYREAN diffractometer with $\mathrm{Cu}-\mathrm{K} \alpha$ radiation. The surface structures of the samples were observed on a scanning electron microscope (SEM, Hitachi S-4800). The amount of copper and iron after each treatment were determined by inductively coupled plasma-optical emission spectroscopy (ICP-OES, Jobin Yvon Horiba, ULTIMA $2 \mathrm{C})$. The thermal conductivity was obtained from the thermal diffusivity and the specific heat measured on a Kyoto Electronics LFA-502 laser flash measurement system and a Netzsch DSC404F3 Pegasus, respectively. The electrical conductivity and the Seebeck coefficient were measured under Ar atmosphere simultaneously on an Ozawa Science RZ2001i apparatus.

\section{Results and Discussion}

\subsection{Nanocomposite Structure}

Figure 1 shows the XRD patterns of the copper ferrites after each treatment. These patterns indicate that the precursor consisted mainly of $\mathrm{CuFe}_{2} \mathrm{O}_{4}$ (Fig. 1(a)), and

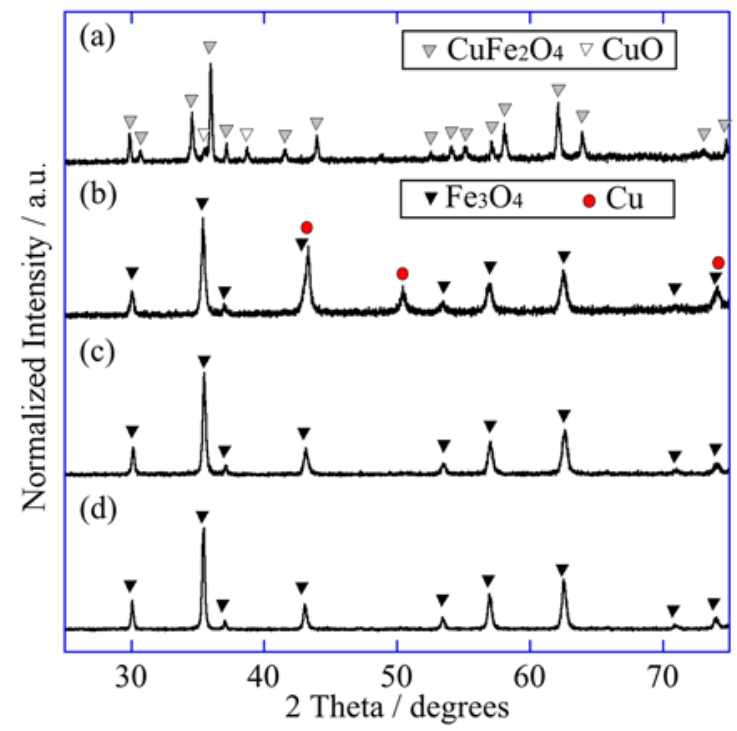

Fig. 1: XRD patterns of $\mathrm{CuFe}_{2} \mathrm{O}_{4}$ (a) before and (b) after reduction, (c) after reduction and leaching for 1 and (d) 5 times.
Table 1. The $\mathrm{Cu}$ amount in the samples after each treatment relative to the nominal $\mathrm{Cu}$ amount in $\mathrm{CuFe}_{2} \mathrm{O}_{4}$.

\begin{tabular}{|l|c|}
\hline Treatment & Relative $\mathrm{Cu}$ amount $/ \mathrm{CuFe}_{2} \mathrm{O}_{4}$ u.f. \\
\hline only reduction & 1.03 \\
\hline 1 R-L cycle & 0.20 \\
\hline 3 R-L cycle & 0.13 \\
\hline 5 R-L cycle & 0.11 \\
\hline
\end{tabular}

metallic $\mathrm{Cu}$ and $\mathrm{Fe}_{3} \mathrm{O}_{4}$ formed after the reduction (Fig. 1(b)). After leaching with $69 \% \mathrm{HNO}_{3}$, the $\mathrm{Cu}$ peaks at 43.3, 50.4 and $74.1^{\circ}$ disappeared (Fig. 1(c)). These results demonstrate that $69 \%$ nitric acid can preferentially remove the metallic $\mathrm{Cu}$ from the reduced $\mathrm{CuFe}_{2} \mathrm{O}_{4}$ leaving $\mathrm{Fe}_{3} \mathrm{O}_{4}$ as a matrix. Table 1 summarizes the results of ICPOES analysis for the samples. The amount of $\mathrm{Cu}$ in the samples after the reducing-leaching (R-L) cycles of 1 and 5 times decreased to $20 \%$ and $11 \%$ of the initially charged $\mathrm{Cu}$, respectively. These results suggest that the copper amount remaining in the $\mathrm{Fe}_{3} \mathrm{O}_{4}$ matrix can be controlled by the R-L cycles.

Figure 2 shows the surfaces of the samples after each treatment. Whereas the surface of the copper spinel before the reduction is dense and smooth (Fig. 2(a)), a number of $\mathrm{Cu}$ nanoparticles were observed on the surface of the reduced $\mathrm{CuFe}_{2} \mathrm{O}_{4}$ (Fig. 2(b)). A nanoporous structure formed after leaching $\mathrm{Cu}$ nanoparticles (Fig. 2(c)), and the pore diameter was similar to the size of the $\mathrm{Cu}$ nanoparticles observed prior to the leaching treatment. These results indicate that the nanoporous structure was formed by selective leaching of copper nanoparticles from the reduced $\mathrm{CuFe}_{2} \mathrm{O}_{4}$. After the R-L cycles for 5 times, the pore size increased, from $50 \mathrm{~nm}$ to more than $100 \mathrm{~nm}$ (Fig. 2(d)).

These observation suggests that the structural transformation proceeds as depicted in Fig. 3. The nanoporous
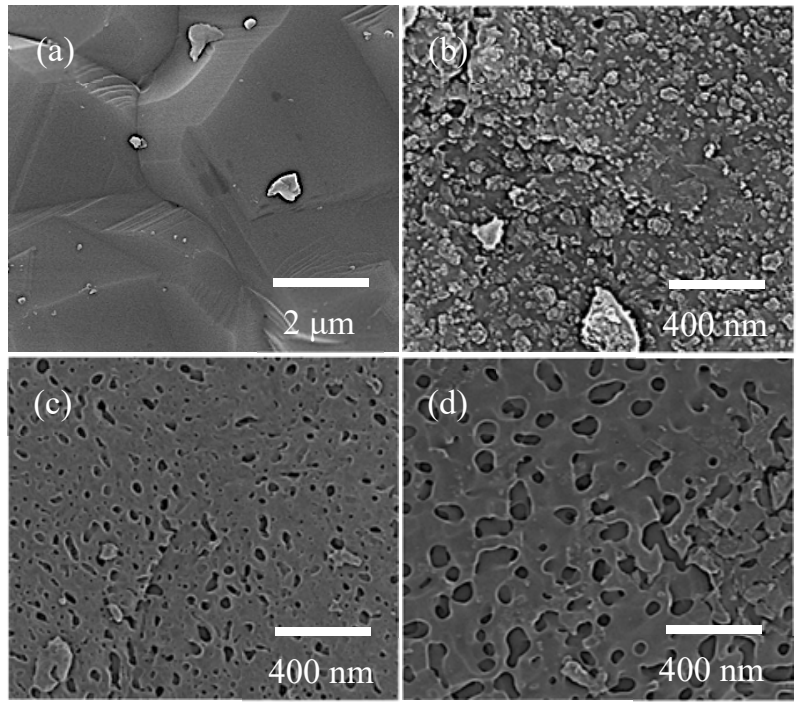

Fig. 2: SEM images of $\mathrm{CuFe}_{2} \mathrm{O}_{4}$ (a) before and (b) after reduction, (c) after reduction and leaching for 1 and (d) for 5 times. 


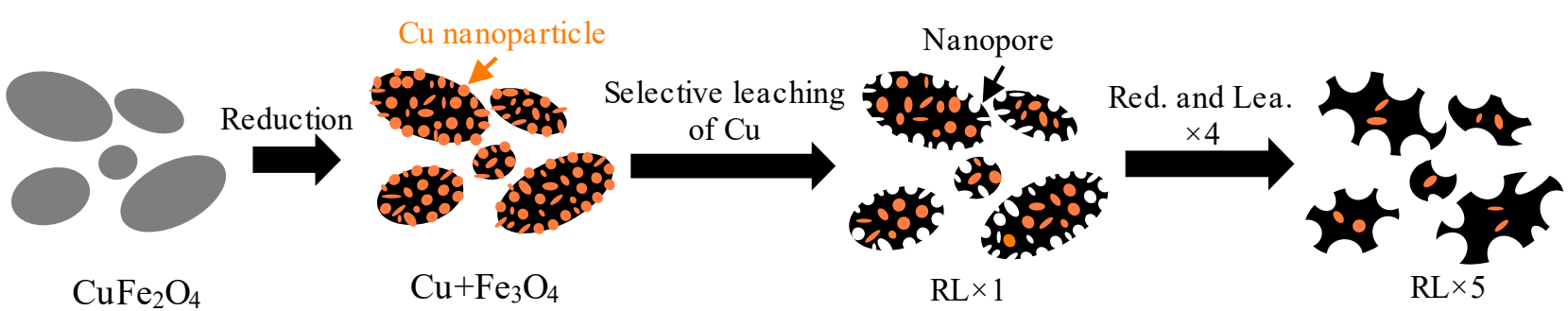

Fig. 3: Schematic diagram for synthesis of nanoporous $\mathrm{Fe}_{3} \mathrm{O}_{4}$ containing $\mathrm{Cu}$ nanoparticles.

$\mathrm{Fe}_{3} \mathrm{O}_{4}$ composite powders containing $\mathrm{Cu}$ nanoparticles were thereby synthesized by selective leaching of the $\mathrm{Cu}$ nanoparticles from the reduced $\mathrm{CuFe}_{2} \mathrm{O}_{4}$.

\subsection{Thermoelectric properties}

In order to investigate thermoelectric properties of the $\mathrm{Fe}_{3} \mathrm{O}_{4}$ nanocomposites thus obtained, we sintered the nanocomposite powders at $1270{ }^{\circ} \mathrm{C}$ in $\mathrm{N}_{2}$. Sintered bodies of the $\mathrm{Fe}_{3} \mathrm{O}_{4}$ nanocomposites after 1 and $5 \mathrm{R}$-L cycles will be denoted as $1 \mathrm{RL}$ and $5 \mathrm{RL}$, respectively. $\mathrm{Fe}_{3} \mathrm{O}_{4}$ represents the reagent grade $\mathrm{Fe}_{3} \mathrm{O}_{4}$ sintered under the same conditions. The relative densities of $1 \mathrm{RL}, 5 \mathrm{RL}$, and $\mathrm{Fe}_{3} \mathrm{O}_{4}$ were 96,94 , and $99 \%$, respectively. The nanoporous structure disappeared from the surface of the sintered bodies (Fig. 4), while the main phase of the samples remained $\mathrm{Fe}_{3} \mathrm{O}_{4}$ with a small XRD peak of $\mathrm{Cu}$ at $2 \theta=50.4$ ${ }^{\circ}$ (Fig. 5), being consistent with the ICP results (Table 1). Therefore, it is plausible that the $\mathrm{Cu}$ nanoparticles

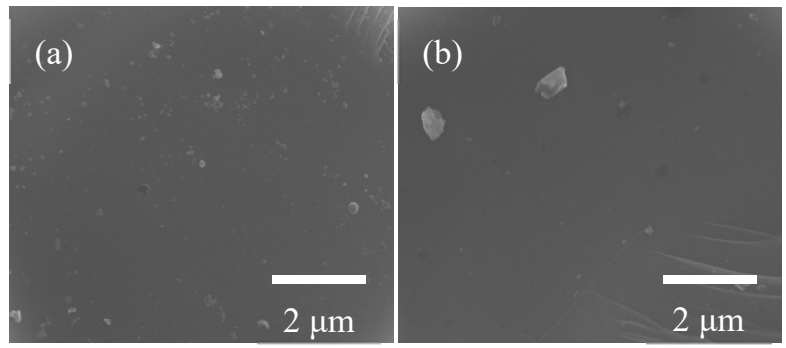

Fig. 4: SEM images of the surfaces of (a) $1 \mathrm{RL}$ and (b) 5RL. remaining in the $\mathrm{Fe}_{3} \mathrm{O}_{4}$ matrix would influence the thermoelectric properties.

Figure 6 shows the temperature dependence of the electrical conductivity, $\sigma$. All the samples exhibited the semiconducting behavior, in which $\sigma$ increases with increasing temperature. The $\sigma$ values of $1 \mathrm{RL}$ and $5 \mathrm{RL}$ were about 10 times higher than that of $\mathrm{Fe}_{3} \mathrm{O}_{4}$ without $\mathrm{Cu}$. Since the $\mathrm{CuFe}_{2} \mathrm{O}_{4}$ phase is much less conductive $\left(\sim 10^{-1}\right.$ $\mathrm{S} \mathrm{cm}-1$ at $300{ }^{\circ} \mathrm{C}$ ) than $\mathrm{Fe}_{3} \mathrm{O}_{4},{ }^{27)}$ even if $\mathrm{CuFe}_{2} \mathrm{O}_{4}$ remained in the samples, it cannot rationalize the much higher $\sigma$ of 1RL and 5RL. Therefore, only possible reason of the markedly increased $\sigma$ would be the metallic $\mathrm{Cu}$ nanoparticles remaining in the $\mathrm{Fe}_{3} \mathrm{O}_{4}$ matrix. Figure 7 shows the temperature dependence of the Seebeck coefficient, $S$, which was almost the same for all the samples. This implies that the $\mathrm{Cu}$ nanoparticles remaining in the matrix has negligible influence on $S$.

The electrical conductivity, $\sigma$, is given by the following equation:

$$
\sigma=n e \mu
$$

where $n, e$, and $\mu$ are the carrier concentration, electrical charge of the carrier, and the carrier mobility, respectively. For degenerate semiconductors, the Seebeck coefficient is given by:

$$
S=\frac{8 \pi^{2} k_{\mathrm{B}}^{2}}{3 e h^{2}} m^{*} T\left(\frac{\pi}{3 n}\right)^{2 / 3} \propto n^{-2 / 3}
$$
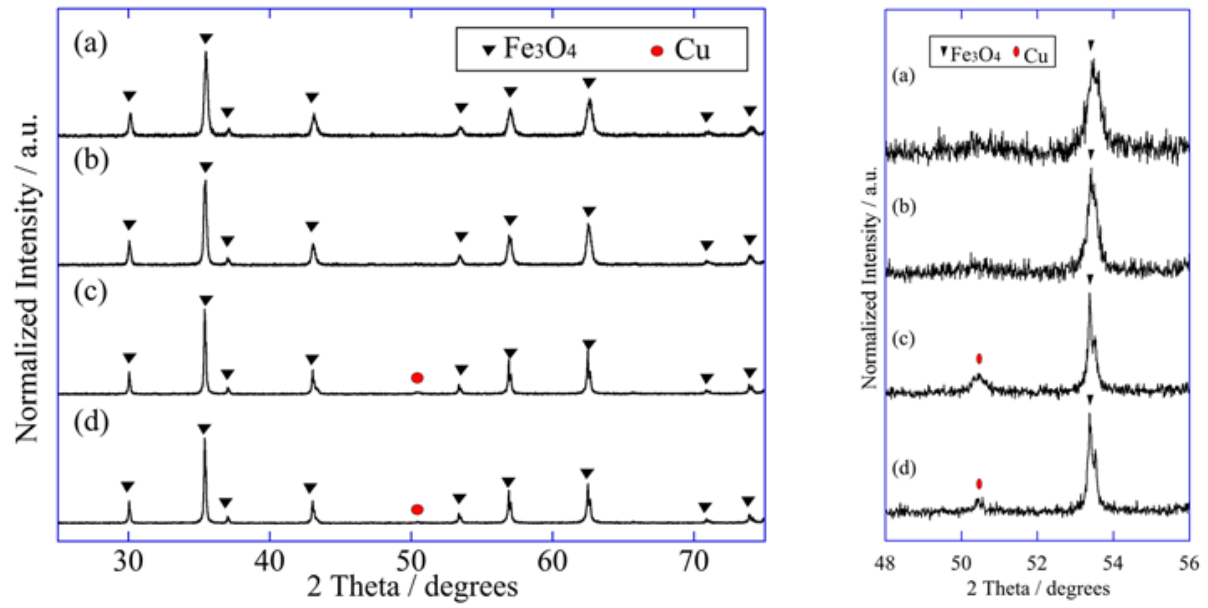

Fig. 5: XRD patterns for $1 R L$ and 5RL $(a, b)$ before and (c, d) after sintering. 


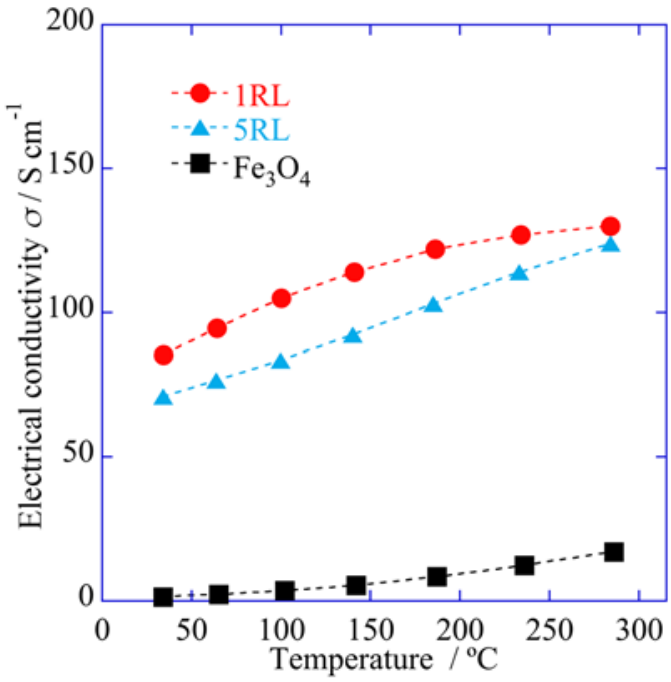

Fig. 6: Temperature dependence of the electrical conductivity of the samples.

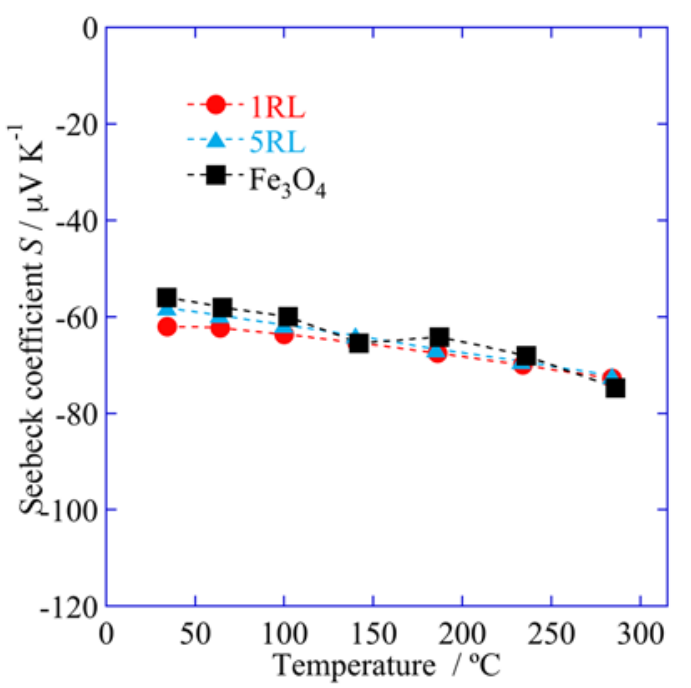

Fig. 7: Temperature dependence of the Seebeck coefficient of the samples.

where $k_{\mathrm{B}}, h$, and $m^{*}$ are the Boltzmann constant, the Plank constant, and the effective mass of the carrier, respectively. These equations show that $\sigma$ and $S$ are oppositely dependent on $n$, while our results are inconsistent with this relation. It is therefore implied that the $\mathrm{Cu}$ nanoparticles provide an additional conduction path with higher carrier mobility, giving negligible influence on the overall carrier concentration.

Figure 8 shows the temperature dependence of the thermal conductivity of the samples. The thermal conductivity of the samples containing $\mathrm{Cu}$ was significantly lower than that of the $\mathrm{Cu}$-free $\mathrm{Fe}_{3} \mathrm{O}_{4}$. Moreover, the more $\mathrm{Cu}$ remained in the samples, the more $\kappa$ decreased. To investigate the carrier and phonon thermal conduction, we calculated the electron thermal conductivity, $\kappa_{\mathrm{el}}$, and the phonon thermal conductivity, $\kappa_{\mathrm{ph}}$, using the following equations:

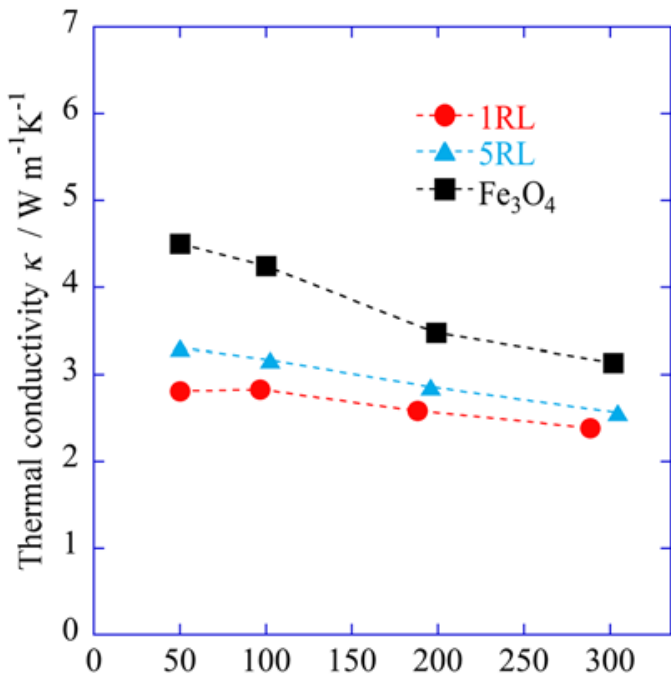

Fig. 8: Temperature dependence of the thermal conductivity of the samples.

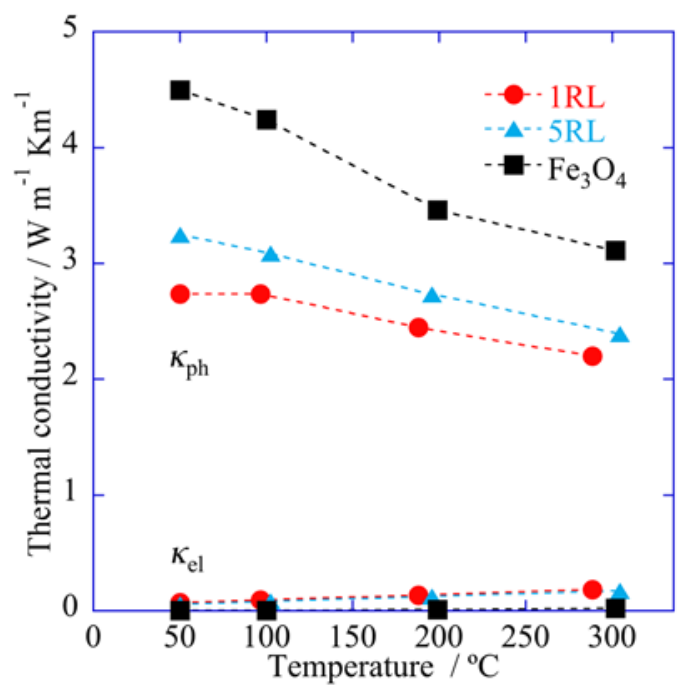

Fig. 9: Temperature dependence of the electron and phonon thermal conductivity of the samples.

$$
\kappa_{\mathrm{ph}}=\kappa-\kappa_{\mathrm{el}},
$$

where $L_{0}$ is the Lorentz number, $2.45 \times 10^{-8} \mathrm{~V}^{2} \mathrm{~K}^{-2}$. Equation (4) is the Wiedemann-Franz law. Figure 9 shows the temperature dependence of $\kappa_{\mathrm{el}}$ and $\kappa_{\mathrm{ph}}$ of the samples. The electron thermal conductivity of all samples was very small. Although $\kappa_{\mathrm{el}}$ of $1 \mathrm{RL}$ and 5RL was slightly higher than that of $\mathrm{Cu}$-free $\mathrm{Fe}_{3} \mathrm{O}_{4}$, the maximum $\kappa_{\mathrm{el}}$ was just 0.20 $\mathrm{W} \mathrm{m} \mathrm{m}^{-1} \mathrm{~K}^{-1}$ at $289{ }^{\circ} \mathrm{C}$ for $1 \mathrm{RL}$. On the other hand, $\kappa_{\mathrm{ph}}$ is strongly dependent on the $\mathrm{Cu}$ amount remaining after the leaching. The $\kappa_{\mathrm{ph}}$ values substantially decreased with increasing $\mathrm{Cu}$ amount, and the lowest $\kappa_{\mathrm{ph}}$ was obtained for $1 \mathrm{RL}$ containing $20 \%$ of initial amount of $\mathrm{Cu}$. These results clearly indicated that the $\mathrm{Cu}$ nanoparticles remaining in the $\mathrm{Fe}_{3} \mathrm{O}_{4}$ matrix increased $\sigma$ (and hence $\kappa_{\mathrm{el}}$ ), and decreased $\kappa_{\mathrm{ph}}$ simultaneously, successfully decoupling electrical and thermal conductions.

In order to evaluate influence of the $\mathrm{Cu}$ nanoparticles on the thermoelectric properties, we have 


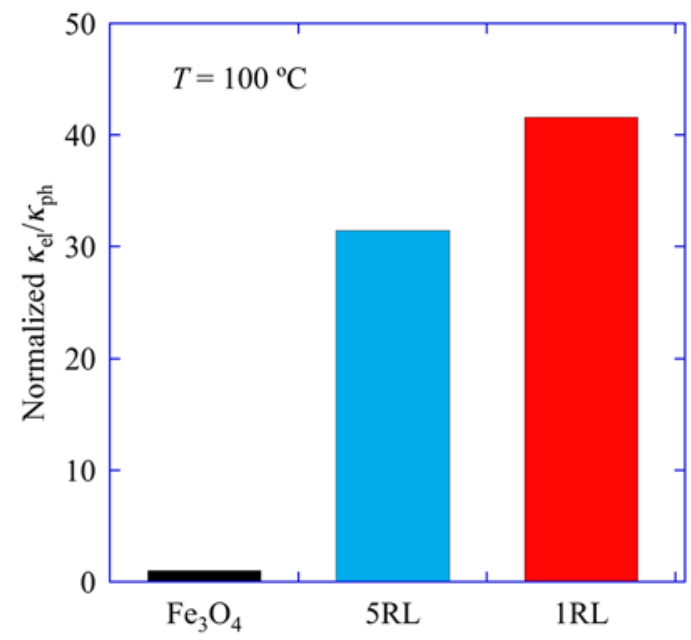

Fig. 10: The normalized $\kappa_{\mathrm{el}} / \kappa_{\mathrm{ph}}$ ratios of the samples at $100^{\circ} \mathrm{C}$.

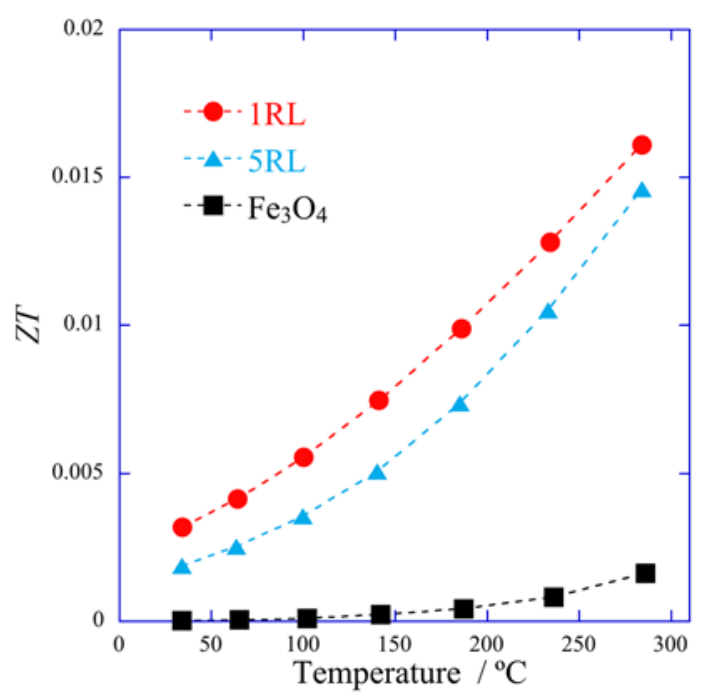

Fig. 11: Temperature dependence of dimensionless figure-ofmerit of the samples.

examined a ratio $\kappa_{\mathrm{e}} / \kappa_{\mathrm{ph}}$ as a quantitative measure. From eq. (1), (4) and (5), the definition of the dimensionless figure-of-merit, $Z T$, yields the following relation when $\kappa_{\mathrm{el}} \ll<\kappa_{\mathrm{ph}}$ :

$$
Z T=\frac{S^{2} \sigma T}{\kappa_{\mathrm{el}}+\kappa_{\mathrm{ph}}}=\frac{\frac{S^{2}}{L_{0}}}{1+\frac{\kappa_{\mathrm{ph}}}{\kappa_{\mathrm{el}}}} \sim \frac{S^{2}}{L_{0}} \frac{\kappa_{\mathrm{el}}}{\kappa_{\mathrm{ph}}} .
$$

As shown in eq. (6), if $\kappa_{\mathrm{el}} \ll \kappa_{\mathrm{ph}}$ and $S$ is almost unchanged, we can use the ratio, $\kappa_{\mathrm{el}} / \kappa_{\mathrm{ph}}$, as an indicator of selective phonon scattering or decoupling of the electron and phonon conductions. Figure 10 compares the $\kappa_{\mathrm{el}} / \kappa_{\mathrm{ph}}$ ratios at $100{ }^{\circ} \mathrm{C}$ normalized by that of $\mathrm{Fe}_{3} \mathrm{O}_{4}$, and indicates that the samples containing the $\mathrm{Cu}$ nanoparticles have more than 30 times larger $\kappa_{\mathrm{el}} / \kappa_{\mathrm{ph}}$. It is therefore presumable that the $\mathrm{Cu}$ nanoparticles in the $\mathrm{Fe}_{3} \mathrm{O}_{4}$ matrix can simultaneously enhance the electron conduction and suppress the phonon conduction.
Figure 11 shows the temperature dependence of the dimensionless figure-of-merit of the samples. The highest $Z T$ value of $1.57 \times 10^{-2}$ at $284^{\circ} \mathrm{C}$ obtained for $1 \mathrm{RL}$ was 10 times larger than that of $\mathrm{Fe}_{3} \mathrm{O}_{4}$. The normalized $\kappa_{\mathrm{e}} / \kappa_{\mathrm{ph}}$ ratio of $1 \mathrm{RL}$ at $284{ }^{\circ} \mathrm{C}$ was 11.4 , confirming that the prediction by eq. (6) is in good accordance with the observed increase in $Z T$. Since the relative density, $d_{\text {rel }}$, of the sintered samples were all more than $94 \%$, and $\kappa_{\text {ph }}$ was substantially lower for 1 RL with $d_{\text {rel }}=96 \%$ than that of $5 \mathrm{RL}$ with $d_{\text {rel }}=94 \%$, the relative density or porosity of the samples is unable to rationalize the large difference in $\kappa_{\text {ph }}$. The most plausible explanation would be the fact that 1RL contained $20 \%$ of initial amount of $\mathrm{Cu}$ presumably as nanoparticles, which increased $\sigma$ by providing a favorable conduction path and decreased $\kappa_{\mathrm{ph}}$ by enhancing phonon scattering simultaneously. It is hence strongly suggested that optimization of the amount of the $\mathrm{Cu}$ nanoparticles enables us to improve the thermoelectric properties of the oxide further. Moreover, if the nanoporous structure of the powders after the R-L cycles can be preserved during sintering, the thermal conductivity would further decrease, improving $Z T$ furthermore.

\section{Conclusions}

Nanoporous $\mathrm{Fe}_{3} \mathrm{O}_{4}$ composite powders containing $\mathrm{Cu}$ nanoparticles were synthesized by selective leaching of the $\mathrm{Cu}$ nanoparticles from reduced $\mathrm{CuFe}_{2} \mathrm{O}_{4}$ matrix. The porous structure and the amount of $\mathrm{Cu}$ remaining in the $\mathrm{Fe}_{3} \mathrm{O}_{4}$ matrix can be controlled by reducing-leaching cycles. After sintering, the porous structure disappeared, whereas the left-over $\mathrm{Cu}$ nanoparticles improve the thermoelectric properties. The more $\mathrm{Cu}$ remained in the $\mathrm{Fe}_{3} \mathrm{O}_{4}$ matrix, the higher electrical conductivity and the lower thermal conductivity were obtained. These results suggested that the $\mathrm{Cu}$ nanoparticles remaining in the $\mathrm{Fe}_{3} \mathrm{O}_{4}$ improved the thermoelectric properties by the provision of carrier conduction path and the enhancement of phonon scattering, making the $Z T$ about 10 times higher than that of the $\mathrm{Fe}_{3} \mathrm{O}_{4}$ without $\mathrm{Cu}$. Furthermore, all the results mean that we therefore established nanostructurecontrol approach to increase electrical conductivity and decrease thermal conductivity simultaneously. We can expect an introduction of nanocomposite structure to some good thermoelectric materials such as $\mathrm{SrTiO}_{3}$ and $\mathrm{ZnO}$ by using this approach, and improve their thermoelectric properties further.

\section{Acknowledgements}

The authors would like to thank Assoc. Prof. K. Suekuni and Assist. Prof. K. Watanabe for their valuable discussion, and Fukuoka Industrial Technology Center for XRD measurement and SEM observation.

This work was financially supported by JSPS KAKENHI Grant Number JP19H02800. 


\section{References}

1) A.M.M. Ismaiel, S.M. Metwalli, B.M.N. Elhadidi, and S. Yoshida, Evergreen, 4, 1 (2017).

2) P. Pal, A.K. Nayak, and R. Dev, Evergreen, 5, 52 (2018).

3) C.J. Vineis, A. Shakouri, A. Majumdar, and M.G. Kanatzidis, Adv. Mater., 22, 3970 (2010).

4) B. Xu, T. Feng, M.T. Agne, L. Zhou, X. Ruan, G.J. Snyder, and Y. Wu, Angew. Chemie - Int. Ed., 56, 3546 (2017).

5) K. Biswas, J. He, Q. Zhang, G. Wang, C. Uher, V.P. Dravid, and M.G. Kanatzidis, Nat. Chem., 3, 160 (2011).

6) K. Biswas, J. He, I.D. Blum, C.I. Wu, T.P. Hogan, D.N. Seidman, V.P. Dravid, and M.G. Kanatzidis, Nature, 489, 414 (2012).

7) T. Mori, Small, 13, 1 (2017).

8) G. Joshi, H. Lee, Y. Lan, X. Wang, G. Zhu, D. Wang, R.W. Gould, D.C. Cuff, M.Y. Tang, M.S. Dresselhaus, G. Chen, and Z. Ren, Nano Lett., 8, 4670 (2008).

9) B. Poudel, Q. Hao, Y. Ma, Y. Lan, A. Minnich, B. Yu, X. Yan, D. Wang, A. Muto, D. Vashaee, X. Chen, J. Liu, M.S. Dresselhaus, G. Chen, and Z. Ren, Science, 320, 634 (2008).

10) G. Rogl, A. Grytsiv, P. Rogl, N. Peranio, E. Bauer, M. Zehetbauer, and O. Eibl, Acta Mater, 63, 30 (2014).

11) G. Rogl, A. Grytsiv, K. Yubuta, S. Puchegger, E. Bauer, C. Raju, R.C. Mallik, and P. Rogl, Acta Mater., 95, 201 (2015).

12) S. Grasso, N. Tsujii, Q. Jiang, J. Khaliq, S. Maruyama, M. Miranda, K. Simpson, T. Mori, and M.J. Reece, $J$. Mater. Chem. C, 1, (12) 2362 (2013).

13) L. Hu, T. Zhu, X. Liu, and X. Zhao, Adv. Funct. Mater., 24, 5211 (2014).

14) L.P. Hu, T.J. Zhu, Y.G. Wang, H.H. Xie, Z.J. Xu, and X.B. Zhao, NPG Asia Mater, 6, 1 (2014).

15) Z.J. Xu, L.P. Hu, P.J. Ying, X.B. Zhao, and T.J. Zhu, Acta Mater, 84, 385 (2015).

16) J. He, J.R. Sootsman, S.N. Girard, J.C. Zheng, J. Wen, Y. Zhu, M.G. Kanatzidis, and V.P. Dravid, J. Am. Chem. Soc., 132, 8669 (2010).

17) J.F. Li, W.S. Liu, L.D. Zhao, and M. Zhou, NPG Asia Mater, 2, 152 (2010).

18) C. Nethravathi, C.R. Rajamathi, M. Rajamathi, R. Maki, T. Mori, D. Golberg, and Y. Bando, J. Mater. Chem. A, 2, 985 (2014).

19) X. Shi, J. Yang, J.R. Salvador, M. Chi, J.Y. Cho, H. Wang, S. Bai, J. Yang, W. Zhang, and L. Chen, J. Am. Chem. Soc., 133, 7837 (2011).

20) T. Liang, X. Su, Y. Yan, G. Zheng, Q. Zhang, H. Chi, X. Tang, and C. Uher, J. Mater. Chem. A, 2, 17914 (2014).

21) M.G. Kanatzidis, Chem. Mater., 22, 648 (2010).

22) M. Ohtaki, and K. Araki, J. Ceram. Soc. Japan, 119, 813 (2011).

23) H. Machrafi, and G. Lebon, Phys. Lett. Sect. A Gen.
At. Solid State Phys., 379, 968 (2015).

24) Y.H. Huang, S.F. Wang, A.P. Tsai, and S. Kameoka, Ceram. Int., 40, 4541 (2014).

25) S. Kameoka, T. Tanabe, and A.P. Tsai, J. Ceram. Soc. Japan, 22, 138 (2010).

26) Y.H. Huang, S.F. Wang, A.P. Tsai, and S. Kameoka, $J$. Power Sources, 281, 138 (2015).

27) A.I. Eatah, A.A. Ghani, and E. El Faramawy, Phys. Status Solidi, 105, 231 (1988). 